\title{
The crystal chemistry of sakuraiite
}

Koichi Momma $^{1}$, Ritsuro Miyawaki ${ }^{1}$, Satoshi Matsubara ${ }^{1}$, Masako Shigeoka ${ }^{1}$, Toshiro Nagase ${ }^{2}$, Seiji Kamada ${ }^{2}$, Shin Ozawa ${ }^{2}$, Eiji Ohtani $^{2}$, Masaaki Shimizu ${ }^{3}$, Akira Kato ${ }^{1}$

${ }^{1}$ National Museum of Nature And Science, Tsukuba, Japan, ${ }^{2}$ Tohoku University, Sendai, Japan, ${ }^{3}$ University of Toyama, Toyama, Japan

E-mail: k-momma@kahaku.go.jp

Sakuraiite is an In-bearing sulfide mineral discovered from Ikuno mine, Hyogo Prefecture, Japan. In the original description [1], sakuraiite was reported as tetragonal (pseudo-cubic with $c=2 a$ ) with an ideal formula of $(\mathrm{Cu}, \mathrm{Zn}, \mathrm{Fe})_{3}(\mathrm{In}, \mathrm{Sn}) \mathrm{S}_{4}$. On the other hand, based on X-ray presession images and chemical analyses, Kissin \& Owens [2] concluded that sakuraiite was cubic phase with an ideal formula of $(\mathrm{Cu}, \mathrm{Zn}, \mathrm{Fe}, \mathrm{In}, \mathrm{Sn}) \mathrm{S}$. The latter interpretation conflicts with the fact that the stoichiometry of sakuraiite is kept constant for a wide-range of compositions by the coupled substitution of $\left(\mathrm{Zn}^{2+}, \mathrm{Fe}^{2+}\right) \mathrm{In}^{3+}$ for $\mathrm{Cu}^{+} \mathrm{Sn}^{4+}$, where the $\mathrm{Cu}$ is monovalent, while in the hypothetical end-member composition of sakuraiite CuS by Kissin \& Owens, Cu should be divalent. In recent years, another problem arose by approval of ishiharaite (Cu, Ga,Fe,In,Zn)S as a distinct new mineral (IMA2013-119) by the commission of the International Mineralogical Association (IMA). Because ishiharaite is reported to have cubic sphalerite-type structure with only one crystalographic site for metal ions [3], the ideal end-member composition of ishiharaite should be defined as CuS, which is ultimately equivalent with the definition of sakuraiite by Kissin \& Owens, i.e., CuS with the cubic sphalerite-type structure. If this is the case one of sakuraiite or ishiharaite should be discredited.

In order to sort out confusion and uncertainty of the definition of sakuraiite, the crystal chemistry of sakuraiite was reinvestigated by single-crystal X-ray diffraction of the type specimen (MSN-M18000) registered in the National Museum of Nature and Science, Tokyo. As was reported by previous studies, fine exsolution lamellae with a variety of compositions (Fig. 1) were observed by electron microprobe. Single-crystal X-ray diffraction experiment on one of the domains having a composition of $(\mathrm{Cu}>\mathrm{Zn}, \mathrm{Fe})$ and (In $>\mathrm{Sn}$ ) revealed a pseudo-cubic crystal structure with a space group $P$-42 $\mathrm{m}$ and $a=$ $5.4500(3) \AA, c=5.4691(3) \AA\left(R_{\text {int }}=2.61 \%, R 1=1.51 \%\right) . \mathrm{Cu}, \mathrm{Zn}$ and Fe atoms are randomly distributed in two crystallographic sites ( $1 a$ and $2 f$ ), while In and $\mathrm{Sn}$ are placed in another site $(1 d)$. This type of metal ordering is different from any other known sulfide structures having sphalerite-type topology, e.g., sphalerite $(F-43 m)$, stannite $(I-42 m)$, kësterite $(I-42 m$ or $I-4)$, and chalcopyrite $(I-42 d)$.

Based on the crystal structure, the ideal formula of sakuraiite should be written as $(\mathrm{Cu}, \mathrm{Zn}, \mathrm{Fe})_{3}(\mathrm{In}, \mathrm{Sn}) \mathrm{S}_{4}$ and it can be considered as one member in a solid solution series of $\mathrm{Cu}_{2}(\mathrm{Zn}, \mathrm{Fe}) \mathrm{SnS}_{4}$ and $\mathrm{Zn}_{2} \mathrm{CuInS}_{4}$.

[1] Kato A (1965) Chigaku Kenkyu 16, 1-7.

[2] Kissin, S. A., \& Owens, D. R. (1986). Canadian Mineralogist, 24, 679-683.

[3] Márquez-Zavalía M F, Galliski M Á, Drábek M, Vymazalová A, Watanabe Y, Murakami H, Bernhardt H J (2014) Canadian Mineralogist 52, 969-980

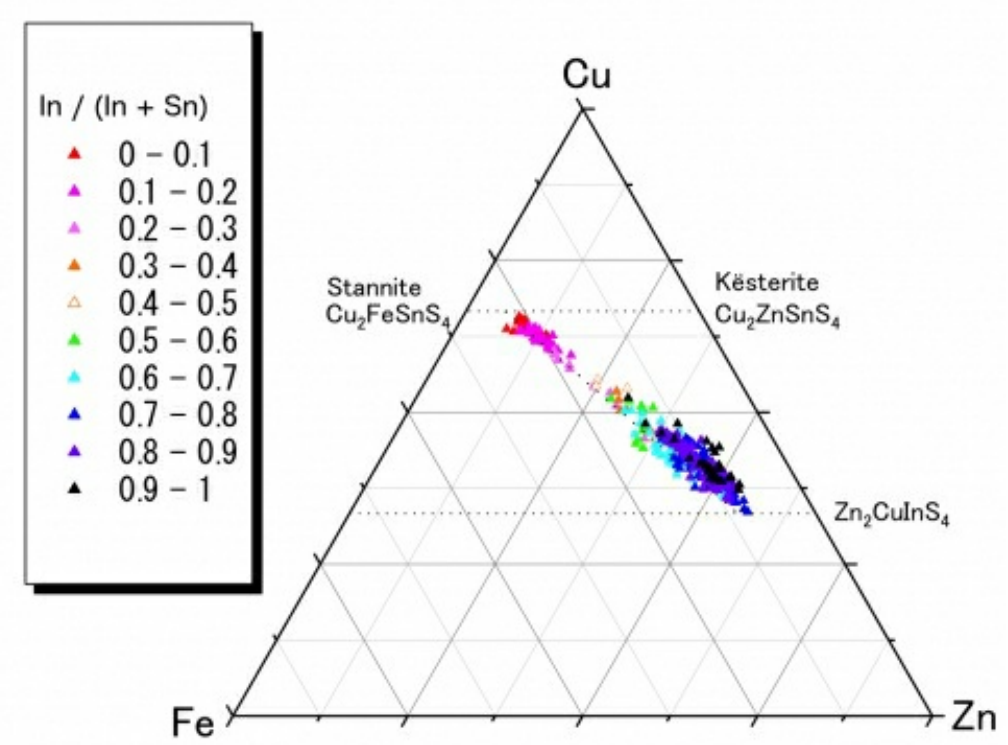

Keywords: sakuraiite, stannite group minerals, solid solution 